\title{
The Multidisciplinary Facets of Research on Humour
}

\author{
Rada Mihalcea ${ }^{1,2}$ \\ ${ }^{1}$ Computer Science Department, University of North Texas \\ ${ }^{2}$ Computational Linguistics Group, Oxford University \\ rada@cs.unt.edu
}

\section{Introduction}

Humour is one of the most interesting and puzzling aspects of human behaviour, and it has been rightfully argued that it plays an important role in an individual's development, as well as in interpersonal communication. Research on this topic has received a significant amount of attention from fields as diverse as linguistics, philosophy, psychology and sociology, and recent years have also seen attempts to build computational models for humour generation and recognition.

In this paper, we summarize the main theories of humour that emerged from philosophical and modern psychological research, and survey the past and present developments in the fields of theoretical and computational linguistics. We also briefly overview related research work in the fields of psychology, sociology and neuroscience. The paper concludes with an illustration of the multidisciplinary applications of humour.

Note that we focus mainly on "verbal humour," which is the most tangible and perhaps the most widely researched form of humour. Although other forms of humour (e.g., visual or situational) have also received attention from the research community, we concentrate our work and consequently this survey on the linguistic expressions of humour.

\section{Theories of Humour}

There are three main theories of humour, which emerged primarily from philosophical studies and research in psychology.

\subsection{Incongruity Theory}

The incongruity theory suggests that humour is due to the mixing of two disparate interpretation frames in one statement. One of the earliest references to an incongruity theory of humour is perhaps due to Aristotle [1 who found that the contrast between expectation and actual outcome is often a source of humour. He is also making a distinction between surprise and incongruity, where the later is presumed to have a resolution that was initially hidden from the audience. The incongruity theory has also found a supporter in Schopenhauer 
[32, who emphasizes the element of surprise by suggesting that "the greater and more unexpected [...] the incongruity is, the more violent will be [the] laughter." The incongruity theory has been formalized as a necessary condition for humour and used as a basis for the Semantic Script-based Theory of Humour (SSTH) [27, and later on the General Theory of Verbal Humour (GTVH) [3].

\subsection{Superiority Theory}

The superiority theory argues that humour is a form of expressing the superiority of one over another. As Hobbes suggested [15, the laughter is "nothing else but sudden glory" triggered by a feeling of superiority with respect to others, or with respect to ourselves in a previous moment. A closely related theory is the one supported by Solomon [33], who suggests that humour is due to feelings of inferiority, which led to the so called "inferiority theory." Although the superiority and inferiority theories of humour have been typically perceived as diametrically opposed, they are in fact intimately related, as the "superior"/"inferior" distinctions are often due to a different point of view. In fact, it can be argued that laughter is triggered by our feelings of superiority with respect to others or ourselves in a previous moment, which are equivalent to feelings of inferiority felt by others or by ourselves in a past moment.

\subsection{Relief Theory}

The third major theory is the relief theory, which suggests that humour is a form of bypassing certain censors that prevent us from having "prohibited thoughts." Eluding these censors results in a release of the energy inhibited by these censors, and consequently the feeling of relief. One of the strongest supporters of the relief theory is Freud [10, who draws a connection between jokes and the unconscious, and Spencer 34, who suggests that laughter is a form of "nervous energy." Some of these ideas have been later embraced by Minsky in his theory of humour [22], to which he adds a cognitive element that attempts to explain the "faulty logic" that is typically encountered in jokes, which is normally suppressed in order to avoid "cognitive harm."

\section{$3 \quad$ Linguistic Research on Humour}

A significant fraction of the research on humour that has been carried out to date has concentrated on the linguistic characteristics of humour. Among the linguistic theories, the most influential is perhaps the General Theory of Verbal Humour (GTVH) 3], which is an extension of the earlier Semantic Script-based Theory of Humour (SSTH) 27].

\subsection{Semantic Script-Based Theory of Humour}

$\mathrm{SSTH}$ is based on the representation of jokes as script opposition, which is an idea closely related to the incongruity resolution theory. Briefly, SSTH defines 
the structure of a joke as consisting of a set-up and a punchline. The set-up has at least two possible interpretations out of which only one is obvious, and consequently the humorous effect is created by the punchline which triggers the second less-obvious interpretation in a surprising way.

The central hypothesis in SSTH is that a text is humorous if the following two conditions are satisfied. First, the humorous text has to be compatible with at least two different interpretations (scripts). And second, the two interpretations have to be opposed to each other. For instance, the following example taken from [27] illustrates this theory: "The first thing that strikes a stranger in New York is a big car." The set-up has two possible interpretations: strike as in "impress" or "hit," which are opposed to each other ("impress" being a positive action, and "hit" triggering negative feelings). The first interpretation is more obvious and thus initially preferred. However, the punchline "by a big car" will change the preference to the second interpretation, which generates the humorous effect.

According to SSTH, the opposition between scripts is binary, and can fall into one of the following three generic types: actual/non-actual, normal/abnormal, possible/impossible, which in turn can be broken down into more specific oppositions, such as positive/negative or good/bad.

\subsection{General Theory of Verbal Humour}

Following SSTH, the GTVH [3] extends the script opposition theory, and adds other possible knowledge resources for a humorous text. While SSTH was primarily focused on semantics, the GTVH is more general and includes other areas in linguistics such as pragmatics and style. GTVH defines six main knowledge resources that can be organized on six levels from concrete (low level) to abstract (high level).

- Script opposition, which is a knowledge source based on the main idea of SSTH of opposing interpretations that are both compatible with the text.

- Logical mechanism, which provides a possible resolution mechanism for the incongruity between scripts.

- Situation, which defines the context of the joke in terms of location, participants, and others.

- Target, which is the person or group of persons that are targeted by the joke.

- Narrative strategy, which defines the style of the joke, i.e., whether it is a dialogue, a riddle or a simple narrative.

- Language, which defines the "surface" of the joke in terms of linguistic aspects such as lexicon, morphology, syntax, semantics.

For example, Attardo and Raskin [3] exemplify the knowledge resources using the following joke: "How many poles does it take to screw in a light bulb? Five. One to hold the light bulb and four to turn the table he is standing on." The script opposition is formed between the expected normal behaviour of a person when screwing in a light bulb, and the "dumb" resolution proposed by the punchline; the logical mechanism is that of "reversal" of a normal behaviour; 
the situation is "bulb changing"; the target of the joke are the "Poles"; and finally, the narrative structure is a "riddle" 28].

An interesting experiment centered around the GTVH theory is reported by Ruch [31, where three jokes are transformed into variants that differed from the original joke in one of the GTVH parameters. A group of 500 subjects were asked to rate the similarity between each of the variants and the original joke on a scale of 1 to 4 . The findings indicate that higher similarity is observed for those variants that differ in a low level parameter in the GTVH hierarchy, thus suggesting that the higher level parameters such as script opposition and logic mechanism are more humour-related 28 .

While GTVH is perhaps the most extensive linguistic theory of humour that has been proposed to date, it has been criticized by Ritchie 28] as lacking theoretical grounds. Ritchie raises doubts about the falsifiability of the GTVH, and about the lack of systematic examples where some of the GTVH knowledge resources are missing, thus resulting in a lack of humorous effect, along with humorous examples that include the missing knowledge resources.

\subsection{Related Work in Linguistics}

Besides the SSTH and the GTVH theories, other research work in linguistics has focused mainly on the analysis of the lexical devices used in humorous text. The syntactic ambiguity often encountered in humour is analysed by Hetzron [14, who describes the structure of jokes and punchlines, and analyses the logical devices found in verbal humour. Oaks 25 is proposing an interesting account on syntactic ambiguity in humour, and identifies several ambiguity "enablers." He focuses mainly on part-of-speech ambiguity, and identifies verbs, articles, and other parts-of-speech that can introduce ambiguity in language (e.g., bite that can be either a verb or a noun).

The lexical and syntactic ambiguity as a source of humour is also studied by Bucaria [6], who analyses the linguistic ambiguity in newspaper headlines. She identifies three main types of ambiguity: lexical (e.g., "Actor sent to jail for not finishing sentence."), syntactic (e.g., "Eye drops off shelf"), and phonological (e.g., "Is there a ring of debris around Uranus"). She also identifies two additional schemata for humorous ambiguity, including the disjunctor/connector model (e.g. "New study on obesity looks for larger group."), and the double ambiguity model (e.g., "Farmer Bill dies in house."). In an analysis of 135 headlines, the lexical and syntactic forms of ambiguity were found to be dominant (71 lexical and 63 syntactic), covering a significant fraction of the corpus, and thus providing support for the incongruity theory of humour.

\section{Computational Humour}

While humour is relatively well studied in fields such as theoretical linguistics [2] and psychology 930, to date only a limited number of research contributions have been made toward the construction of computational humour prototypes. 
Most of the computational approaches to date on style classification have focused on the categorization of more traditional literature genres, such as fiction, scitech, legal, and others [16], and much less on creative writings such as humour.

The most systematic effort in this area is perhaps Ritchie's book on the linguistic analysis of jokes, which brings together research on linguistic theories and artificial intelligence. In addition to a comprehensive overview of the main research contributions in humour, Ritchie is also proposing a classification of jokes into propositional and linguistic, and suggests a structural description of the jokes 28 .

Similar to other computational linguistics research carried out on language style, there are two main research directions in computational humour: (1) humour generation, which attempts to build computational models to generate humorous text, and (2) humour recognition, which deals with the problem of identifying humour in natural language.

\subsection{Humour Generation}

One of the first attempts in humour generation is the work described by Binsted and Ritchie [5], where a formal model of semantic and syntactic regularities was devised, underlying some of the simplest types of puns (punning riddles). The model was then exploited in a system called JAPE that was able to automatically generate amusing puns.

Another humour generation project is the HAHAcronym project [35, whose goal was to develop a system able to automatically generate humorous versions of existing acronyms, or to produce a new amusing acronym constrained to be a valid vocabulary word, starting with concepts provided by the user. The comic effect was achieved mainly by exploiting incongruity theories (e.g., finding a religious variation for a technical acronym).

\subsection{Humour Recognition}

There are only a few studies addressing the problem of humour recognition. The study reported in [36] is devoted to the problem of humour comprehension, focusing on a restricted type of wordplays, namely the "Knock-Knock" jokes. The goal of the study was to evaluate to what extent wordplay can be automatically identified in "Knock-Knock" jokes, and if such jokes can be reliably recognized from other non-humorous text. The algorithm was based on automatically extracted structural patterns and on heuristics heavily based on the peculiar structure of this particular type of jokes. While the generic wordplay recognition gave satisfactory results ( $67 \%$ accuracy), the identification of wordplays that had a humorous effect turned out to be significantly more difficult (12\% accuracy).

In our own previous work 2019], humour recognition was formulated as a text classification task, and machine learning algorithms were run on large collections of humorous texts (oneliners or humorous news articles). Both content and 
stylistic features were evaluated, including n-gram models, alliteration, antonymy, and adult slang, with performance figures significantly higher than apriori known baselines.

Another humour recognition study was reported by Purandare and Littman 26], where the recognition experiments were performed using both content features and spoken dialogue prosody features (tempo, energy, and pitch). The experiments were run on dialogues from the TV-series "Friends," with significant improvements observed over the baseline. They also reported a gender study, with the improvement obtained for humour recognition in male dialogues being higher than the one obtained for female dialogues, suggesting perhaps that the humorous features are more prominent for males than for females.

\section{Multidisciplinary Research on Humour}

In addition to the research work in linguistics and the recent efforts in computational linguistics, humour has been also studied in other areas.

\subsection{Sociology}

In sociology, humour has been frequently associated with studies concerned with patterns of communication in different groups. For instance, Duncan 8 shows that cohesive and non-cohesive work groups have different humour patterns, suggesting a correlation between the type of humour practiced in a group and the structure of the group.

Studies have also investigated the association between gender and humour, by analysing the type and role of humour for female, male and mixed groups. Hay 12. used a taxonomy of humour in a gender-oriented analysis, which revealed the preference of women for observational humour and the tendency of male groups for insults and roleplay. Interestingly, a correlation was also observed between the gender of these groups and the function of humour; women groups used humour primarily as a social element, whereas men groups often used it as a means for increasing status. Finally, Hay's study also reported on the association between gender and humour topics, suggesting that women use more frequently humour on topics involving people, while men joke more about politics, computers and work; this observation correlates with recent conclusions drawn in corpus-based gender studies [17.

Another aspect of interest in sociology is the relation between culture and humour. Work in this area has highlighted the relation between cultural background and humour appreciation, showing that the set of values and norms of a culture largely determine the content and style of humour [13. Focused studies have highlighted differences between various cultures, as for instance the study reported in 24], which shows how Arab and Jewish communities developed a different sense of humour explained by their diverse background and different social status. 


\subsection{Psychology}

Humour research in psychology has been mainly concerned with the correlation between humour and individual development. There are several studies that considered the cognitive aspects of humour, and the role that humour can play in infants and children development. For instance, it has been found that humour has an important role in improving text comprehension [38.

Other studies have been concerned with the relation between personality profiles and sense of humour. Along these lines, it has been suggested that extroversion and neuroticism can be predicted from humour perception 23. Similarly, humour was found to be related to other personality characteristics such as simplicity-complexity, intelligence, or mood [29].

\subsection{Neuroscience}

In recent years, given the advances made in brain imaging techniques (fMRI or MEG), researchers have started to investigate the brain activity observed during humour detection and comprehension. Recent research findings suggest that the left and the right hemispheres are both involved in humour appreciation, which is an effect that has been observed in verbal humour as well as visual humour 4. Moreover, studies have also observed the activation of the amygdala and midbrain regions (also known as the "pleasure center"), which is probably due to the pleasurable effect created by humour [37.

It is also worth noting the study reported in 23], which shows connections between gender, personality (i.e., extroversion and neuroticism), and humour appreciation, observed using brain imaging techniques. Such associations have been typically identified through surveys conducted in psychological studies, and the study reported in 23] confirms these previous findings by identifying patterns of brain activity occurring during humour comprehension.

\section{Applications of Humour}

In agreement with its multidisciplinary facets, applications of humour are also found in a variety of domains, including social communication, education, health, human-computer interaction.

A few attempts were made to integrate humour into human-computer interfaces. For instance, the JAPE system [7] was integrated into a natural language robot Elmo [18] - a software agent able to carry out a dialogue that was improved with the ability of formulating humorous replies. However, since the puns used by Elmo were automatically generated, the effect was not overwhelmingly positive, as users seemed to dislike the occasional low-quality humour more than the complete absence of it.

Another attempt is the integration of oneliners into email applications. In our own previous work 21, we created an email client that was automatically adding humorous statements to emails, based on the similarity between the body of the email and the candidate oneliners. A user study conducted around the email 
application revealed that users found the humorous additions to be entertaining and appropriate, and considered adopting the application for their own day-byday use.

Humour has also been used for educational purposes. For instance, the Riddles system [38] was used to improve text comprehension in children 7-11 years old. The software showed riddles to children with reading difficulties, and asked them to discuss their various possible interpretations. The interaction with the system was found to improve the reading comprehension scores for the participating children.

Finally, it is also worth mentioning the humour recommender system 11 which builds a "sense of humour" profile for the users, and consequently recommends new jokes based on the rating received from other users with a similar profile.

\section{Conclusions}

In this paper, we tried to bring together the main research achievements on humour in the fields of theoretical and computational linguistics, as well as in sociology, psychology and neuroscience. We hopefully demonstrated that humour is a highly multidisciplinary field of research. We believe this fact has two important implications. First, it means that research on humour can benefit from the diverse expertise of researchers working in different fields, which will broaden the perspective for understanding the role and the nature of humour. Second, because of its multidisciplinary facets, research on humour can serve as a bridge between different fields of study and consequently contribute to scientific interdisciplinarity.

\section{References}

1. Aristotle. Rhetoric. 350 BC.

2. Attardo, S.: Linguistic Theory of Humor. Mouton de Gruyter, Berlin (1994)

3. Attardo, S., Raskin, V.: Script theory revis(it)ed: Joke similarity and joke representation model. Humor: International Journal of Humor Research 4, 3-4 (1991)

4. Bartolo, A., Benuzzi, F., Nocetti, L., Baraldi, P., Nichelli, P.: Humor comprehension and appreciation: An fmri study. Journal of Cognitive Neuroscience 18 (2006)

5. Binsted, K., Ritchie, G.: Computational rules for punning riddles. Humor 10, 1 (1997)

6. Bucaria, C.: Lexical and syntactic ambiguity as a source of humor. Humor 17, 3 (2004)

7. Cawsey, A., Binsted, K., Jones, R.: An implemented model of punning riddles. In: Conference on Artificial Intelligence,Seattle (1995)

8. Duncan, W.: Perceived humor and social network patterns in a sample of taskoriented groups: A reexamination of prior research. Human Relations 37, 11 (1984)

9. Freud, S.: Der Witz und Seine Beziehung zum Unbewussten. Deutike, Vienna (1905)

10. Freud, S.: Jokes and their relation to the unconscious. Humor. International Journal of Psychoanalysis 9 (1960) 
11. Goldberg, K., Roeder, T., Gupta, D., Perkins, C.: Eigentaste: A constant-time collaborative filtering algorithm. Information Retrieval 4, 2 (2001)

12. Hay, J.: Gender and humour: Beyond a joke. Master's thesis, Victoria University of Wellington (1995)

13. Hertzler, J.: Laughter: A social scientific analysis. Exposition Press, New York (1970)

14. Hetzron, R.: On the structure of punchlines. Humor: International Journal of Humor Research 4, 1 (1991)

15. Hobbes, T.: Human Nature in English Works. Molesworth (1840)

16. Kessler, B., Nunberg, G., Schuetze, H.: Automatic detection of text genre. In: Proceedings of the $35^{\text {th }}$ Annual Meeting of the Association for Computational Linguistics (ACL97) Madrid (July 1997)

17. Liu, H., Mihalcea, R.: Of men, women, and computers: Data-driven gender modeling for improved user interfaces. In: International Conference on Weblogs and Social Media (2007)

18. Loehr, D.: An integration of a pun generator with a natural language robot. In: Colloque Linguistique-Informatique de Montreal (1996)

19. Mihalcea, R., Pulman, S.: Characterizing humour: An exploration of features in humorous texts. In: Proceedings of the Conference on Intelligent Text Processing and Computational Linguistics, Mexico City (2007)

20. Mihalcea, R., Strapparava, C.: Making computers laugh: Investigations in automatic humor recognition. In: Proceedings of the Human Language Technology / Empirical Methods in Natural Language Processing conference, Vancouver (2005)

21. Mihalcea, R., Strapparava, C.: Technologies that make you smile: Adding humor to text-based applications. IEEE Intelligent Systems 21, 5 (2006)

22. Minsky, M.: Jokes and the logic of the cognitive unconscious. Tech. rep., MIT Artificial Intelligence Laboratory (1980)

23. Mobbs, D., Hagan, C., Azim, E., Menon, V., Reiss, A.: Personality predicts activity in reward and emotional regions associated with humor. National Academy of Science 102, 45 (2005)

24. Nevo, O.: Appreciation and production of humor as an expression of aggression. Journal of Cross-Cultural Psychology 15, 2 (1984)

25. Oaks, D.: Creating structural ambiguities in humor: Getting English grammar to cooperate. Humor: International Journal of Humor 7, 4 (1994)

26. Purandare, A., Litman, D.: Humor: Prosody analysis and automatic recognition for $\mathrm{F}^{*} \mathrm{R}^{*} \mathrm{I}^{*} \mathrm{E}^{*} \mathrm{~N}^{*} \mathrm{D}^{*} \mathrm{~S} *$. In: Proceedings of the, Conference on Empirical Methods in Natural Language Processing Sydney, Australia, pp. 208-215 (2006)

27. Raskin, V.: Semantic Mechanisms of Humor. Kluwer Academic Publications (1985)

28. Ritchie, G.: The Linguistic Analysis of Jokes. Routledge, London (2003)

29. Ruch, W.: The Sense of Humor: Explorations of a Personality Characteristic (1998)

30. Ruch, W.: Computers with a personality? lessons to be learned from studies of the psychology of humor. In: Proceedings of the The April Fools Day Workshop on Computational Humour (2002)

31. Ruch, W., Attardo, S., Raskin, V.: Toward an empirical verification of the general theory of verbal humor. Humor: International Journal of Humor Research 6, 2 (1993)

32. Schopenhauer, A.: The World as Will and Idea. Kessinger Publishing Company (1819)

33. Solomon, R.: Ethics and Values in the Information Age. Wadsworth, ch. Are the Three Stooges Funny? Soitainly! (or When is it OK to Laugh?) (2002) 
34. Spencer, H.: The physiology of laughter. Macmillan's Magazine 1 (1860)

35. Stock, O., Strapparava, C.: Getting serious about the development of computational humour. In: Proceedings of the $8^{\text {th }}$ International Joint Conference on Artificial Intelligence (IJCAI-03) Acapulco, Mexico (August 2003)

36. Taylor, J., Mazlack, L.: Computationally recognizing wordplay in jokes. In: Proceedings of CogSci 2004, Chicago (August 2004)

37. Watson, K., Matthews, B., Allman, J.: Brain activation during sight gags and language-dependent humor. Cerebral Cortex 17, 2 (2007)

38. Yuill, N.: A funny thing happened on the way to the classroom: Jokes, riddles and metalinguistic awareness in understanding and improving poor comprehension in children (1997) 